\title{
Microclimate Environmental Assessment and Impact of Mountain City Pedestrian Streets in Summer
}

\author{
Ke Xiong ${ }^{1}$, Zhenjing Yang ${ }^{1,2,3, *}$, and Canhua Cheng $^{1}$ \\ ${ }^{1}$ School of Architecture and Urban Planning Chongqing University, Chongqing 400044, PR China \\ ${ }^{2}$ Chen Qigao Building Science and Technology Research Center, Chongqing 400044, PR China \\ ${ }^{3}$ Key laboratory of new technology for construction of cities in mountain area, ministry of education, Chongqing University, Chongqing \\ 400044, PR China
}

\begin{abstract}
In mountainous cities, walking is an important form of transportation. The microclimate environment of pedestrian streets in summer affects the comfort of pedestrians, especially in hot summer cities. Besides, there are many height differences in mountainous city pedestrian streets, while existing researches of the thermal environment were mainly aimed at plain cities. We used typology to analyze different spatial patterns and tested microclimate of five kinds of streets in the Shanchengxiang of Chongqing. Then the universal thermal climate index (UTCI) was used to evaluate the thermal comfort of different spatial spaces. Firstly, the thermal environment of the mountain city street in summer is extremely uncomfortable and needs to be improved. Secondly, the sky view factor ( SVF ) has a great impact on the street thermal environment. Among all kinds of streets, the one-sided open B-N (SVF $=0.474)$ has the worst thermal environment, with an average UTCI of $44.7^{\circ} \mathrm{C}$. However, the two-sided enclosed $\mathrm{B}_{2}-\mathrm{B}_{2}(\mathrm{SVF}=$ 0.052) represents a better thermal environment, with an average UTCI of $35.5^{\circ} \mathrm{C}$. The $\mathrm{R}^{2}$ value of 0.88 reflects that the linear correlation between UTCI and SVF is larger than that of $\mathrm{H} / \mathrm{W}$, whose $\mathrm{R}^{2}$ value is mere 0.04. Finally, different interfaces and enclosure forms have a great impact on space thermal comfort. This study quantifies the parameters that influence the design of pedestrian streets in mountain cities from the perspective of outdoor microclimate environmental assessment and provide a reference for the sustainable design of regional streets.
\end{abstract}

\section{Introduction}

City is an advanced form of human-inhabited life and a political, economic and cultural center of a country or region [1]. With the continuous acceleration of the urbanization process, the relationship between nature and humans has become increasingly alienated. Global problems such as the greenhouse effect, urban heat island effect, haze and so on have been emerging. Urban climate problems have been highlighted, which has led to the change of the microclimate environment due to human factors in the urban center district [2]. People's outdoor public activities are gradually increasing. Establishing a good ecological environmental system is the most generally beneficial welfare for people's livelihood from the aspect of historical and cultural protection and inheritance, or urban improvement and microclimate environment improvement. It is significant to combine natural environment with the designing process of urban planning and architecture to alleviate the urban climate problems and to promote the construction process of ecological civilization and sustainable development. On October 25, 2019, the public information network of Chongqing municipal government released a document named opinions of the general office of Chongqing Municipal People's Government on strengthening the planning and implementation of historical and cultural protection and inheritance. An idea of trinity city which included landscape, culture and the city was put forward to make Chongqing a national historical and cultural city. The concept aims at developing slope greening and cliff park, reforming micro space and increasing community vitality [3]. Striving to build a city full of history, a street full of feelings (Fig. 1), a footpath full of memory (Fig. 2), a building full of story (Fig. 3) and a cultural environment for the whole city is a prerequisite for the promotion of Chongqing. Chongqing is rich in natural resources and humanistic connotations. People's demand for material and culture is increasingly strong. Therefore, it is an urgent problem to transform the mountain city footpath into a green road for travel, a path for leisure and recreation, a path for nostalgia and memory, and a path for urban experience and landscape.

\footnotetext{
* Corresponding author: young30331@163.com
} 


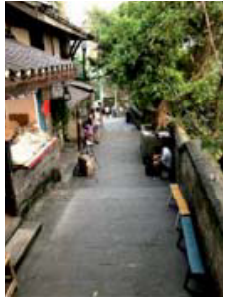

Fig. 1. The street full of feelings

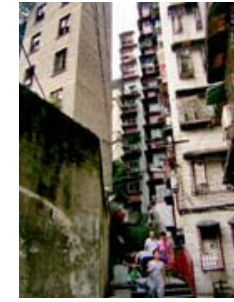

Fig. 2. Trail full of memory

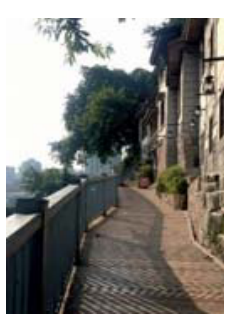

Fig. 3. Architecture full of stories
In recent years, foreign researchers and architects have become more and more interested in urban microclimate. Their researches mainly started from the square, urban street valley and park to analyze the influence of microclimate on human comfort [4]._How to optimize urban space and create a comfortable microclimate environment is also their focus [6-10]. For the past few years, Tan Shaohua's team of Chongqing University has conducted a lot of investigations and researches on the vitality and environment of urban parks and street spaces in Chongqing. A series of guidance suggestions on the construction of urban public space atmosphere and environmental optimization has been proposed. Han Guifeng's team studied the heat island effect and urban interface temperature of mountain cities and put forward a variety of suggestions to improve the comfort of the urban thermal environment [15-20]. However, these studies are generally based either on a macro or medium level of urban thermal environment simulation or qualitative research. This reveals a lack of micro-level research experiments and other quantitative research.

Compared with the architectural climate, the research of urban microclimate is more complex, which includes geography, meteorology, environmental physics, life science, architecture, urban and rural planning, and landscape architecture. This paper mainly tested the thermal environment of the Mountain City Lane and quantified the relationship between microclimate and multiple factors. Finally, it provided scientific guidance for the construction of the ecological civilization of mountain city, as well as designing experience and reference for the construction of livable and healthy living environments of the city in the future.

\section{Methodology}

\subsection{Overview of the research area}

Shanchengxiang, a typical pedestrian street in Yuzhong District, Chongqing, China (Fig. 4), was selected as the research object. Chongqing is located in the southwest part of China, which belongs to the hot summer and cold winter climate zone. The average temperature in the hottest month fluctuates between 26 and 29 . To solve the problem of inconvenient transportation through the mountainous area, a dozen pedestrian routes were planned to link the most attractive public space and surrounding buildings. These routes also combined the green corridor and urban balcony to form a pedestrian terrace connecting the upper and lower half of the city. Among them, Shanchengxiang street (Fig. 5) is one of the representatives of this typical space. It is a traditional folk house with typical mountain features of Bayu area. Built close to the cliff, it also epitomizes Chongqing's history, culture and landscape features. The users are mainly surrounding residents and a small number of tourists [21].

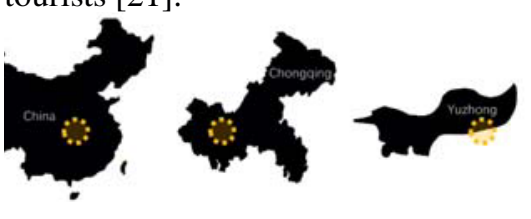

Fig. 4. Geographical location of the research object

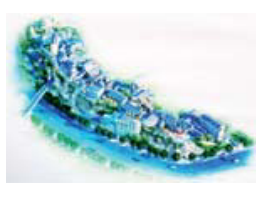

Fig. 5. Rendering of Shanchengxiang

\subsection{Overview of research area Test instruments and methods}

The experiment was conducted between 7:30-19:30 on August 25, 2019. Fig. 6 shows the distributions of measuring points. The measured microclimate parameters include wall temperature, ground temperature, air humidity, wind speed, dry bulb temperature, wet bulb temperature, black bulb temperature and fish eye photo. This experiment adopted the equipment conforming to ISO7726 standard. The time interval for data recording of parameters except fish eye photos was $5 \mathrm{mins}$, and the average value of every 30 mins was taken for analysis. The tested points of temperature, humidity and wind speed were set 1.5 meters above the ground. It should be noted that due to equipment failure, the wind speed at point $\mathrm{E}$ was not recorded. However, the conditions of point $\mathrm{E}$ and point A are similar, both of which are back against the mountain and facing the river. After calculation, the wind speed has little influence on Mean Radiant Temperature(MRT) and Universal Thermal Climate Index(UTCI). Therefore, this study used the wind speed of point A to replace that of point $\mathrm{E}$.

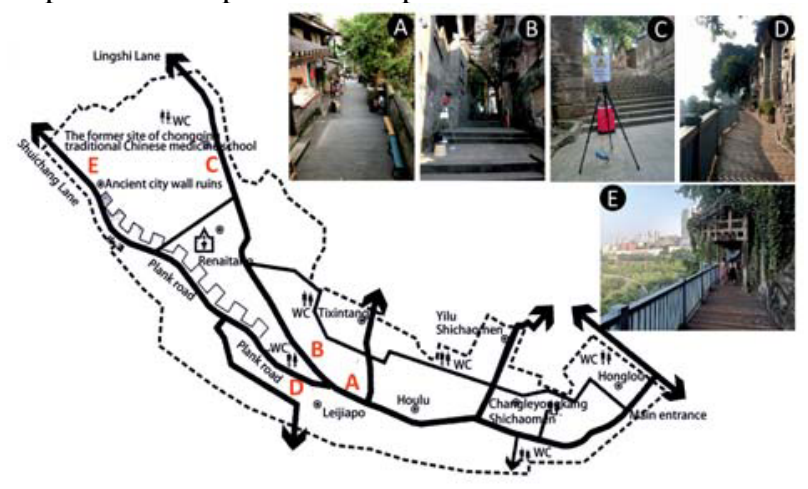

Fig. 6. Distributions of the measuring points

\subsection{Data processing}

Through Ray Man Pro software [22,23], fish eye photos were processed and SVF (sky view factor) of each measured point is calculated. The testing contents of the first group are summarized and shown in Table 1. 
Table 1. Testing contents summary of the first group

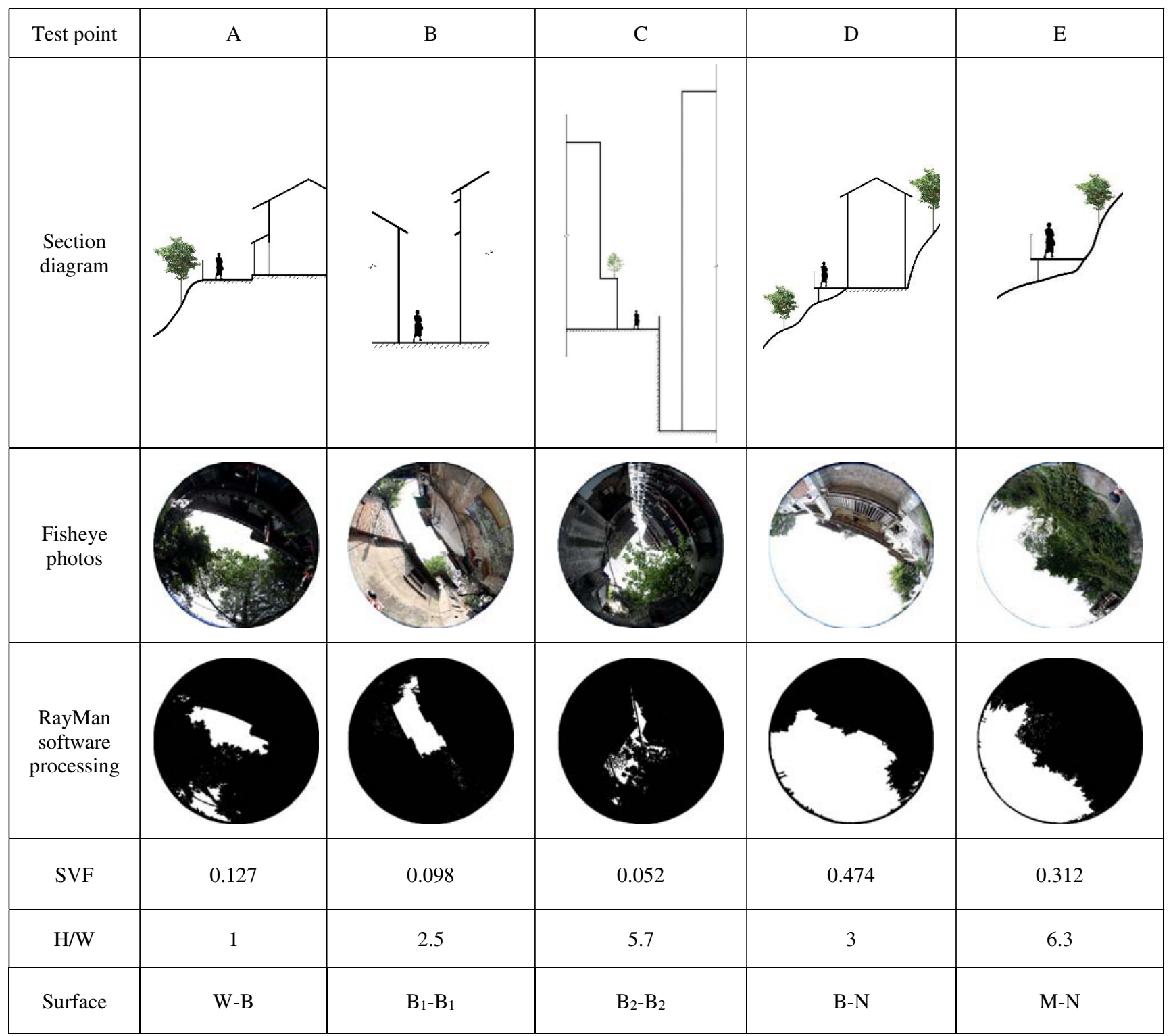

Note: w-b refers to walls and buildings on two sides of the street; $\mathrm{B}_{1}-\mathrm{B}_{1}$ and $\mathrm{B}_{2}-\mathrm{B}_{2}$ refer to buildings on both sides of the street; $\mathrm{B}-\mathrm{N}$ refers to buildings on only one side of the street; $\mathrm{M}-\mathrm{N}$ refers to mountains on only one side of the street.

\section{Results and discussion}

\subsection{Analysis of microclimate environment of mountain city footpath}

We analyzed the air temperature, air humidity, ground temperature and wall temperature of respective tested points. Fig. 7 shows that the temperature distribution is different at each point. The air temperature and wall temperature of point $\mathrm{D}$ and $\mathrm{E}$ both close to the river are very high. Especially at point $\mathrm{D}$, the average temperature exceeds 40 , and the maximum temperature reaches 47.3 . The reason is that one side of both point $\mathrm{D}$ and $\mathrm{E}$ is a building or mountain, and the other side is directly exposed to the sun. The results shows that the ground temperature of point D is extremely high, with the highest temperature reaching 55.5. The temperature fluctuation of point $\mathrm{D}$ is the largest during the testing time, indicating that it is exposed to the sun all the time without shadow. However, the ground temperature of point $\mathrm{A}, \mathrm{B}$ and $\mathrm{C}$ began to drop at 12:00 noon, which suggests that there appeared shadow in the street after 12:00 noon. The humidity trend for each point is basically the same. Nevertheless, due to the high temperature, the relative humidity of point $\mathrm{A}$ is lower than other tested points. The maximum relative humidity of point $\mathrm{A}$ is no more than $60 \%$, and the minimum is only $22.7 \%$. The highest average humidity comes from point $\mathrm{B}$, followed by point $\mathrm{C}$.

\footnotetext{
*Corresponding author: young30331@163.com
} 

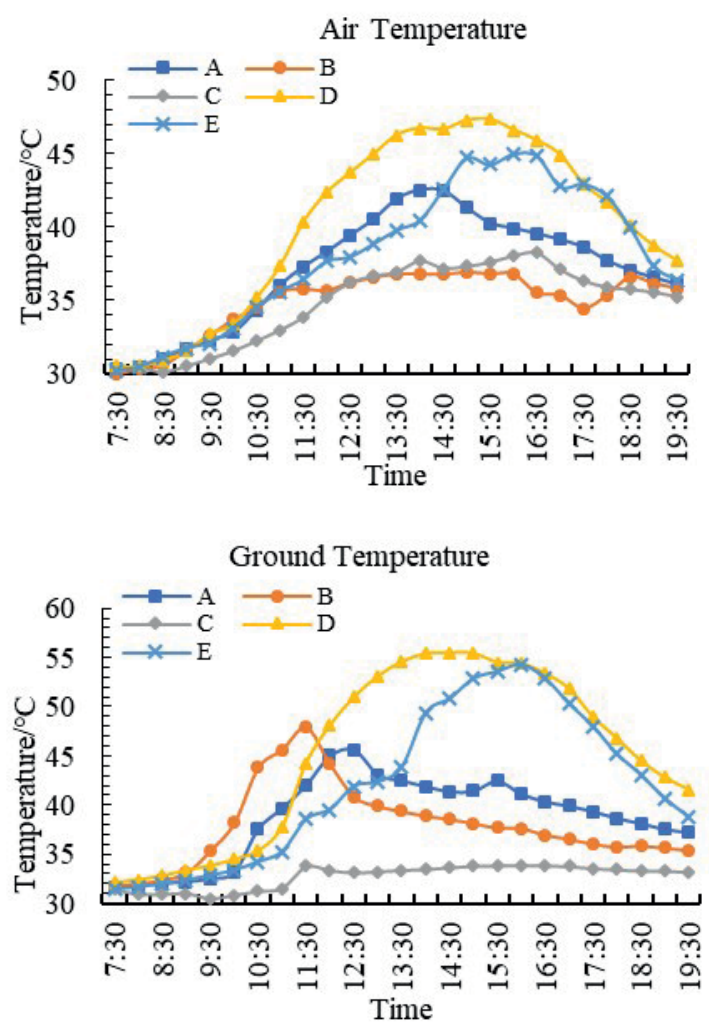

Fig. 7. Microclimate parameters curve of each measuring point

\subsection{Thermal comfort evaluation and analysis of mountain city footpath}

In the outdoor test, the average radiation temperature ( $\left.\mathrm{T}_{\mathrm{mrt}}\right)$ can be approximately calculated from the black ball temperature $\left(\mathrm{T}_{\mathrm{g}}\right)$ and the wind $\operatorname{speed}\left(\mathrm{V}_{\mathrm{a}}\right)$ through the formula[24]. The calculation is shown in formula (1):

$T_{m r t}=\left[(T g+273.15)^{4}+\frac{1.1 * 10^{8} * V_{a}^{0.6}}{\varepsilon * D^{0.4}}\left(T_{g}-T_{a}\right)\right]^{0.25}-273.15$

$\varepsilon$ : Emissivity of black bulb thermometer;

D: Diameter of black bulb thermometer, $\mathrm{mm}$; Diameter of black bulb in this study is $150 \mathrm{~mm}$.

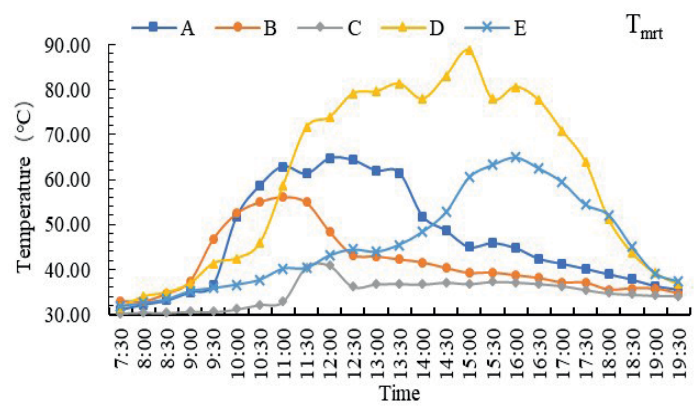

Fig. 8. $\mathrm{T}_{\text {mrt }}$ of each measuring point

From Fig. 8, we can see that $\mathrm{T}_{\mathrm{mrt}}$ of point $\mathrm{D}$ is higher than that of other measuring points during the period of 11:30-18:00. The maximum value of $\mathrm{T}_{\mathrm{mrt}}$ at point $\mathrm{D}$ reached 88.7 , and the time over 70 lasted for 4 hours. The highest value of $\mathrm{T}_{\mathrm{mrt}}$ at point $\mathrm{A}$ and $\mathrm{E}$ appeared after 15:00, while the highest temperature at the other three
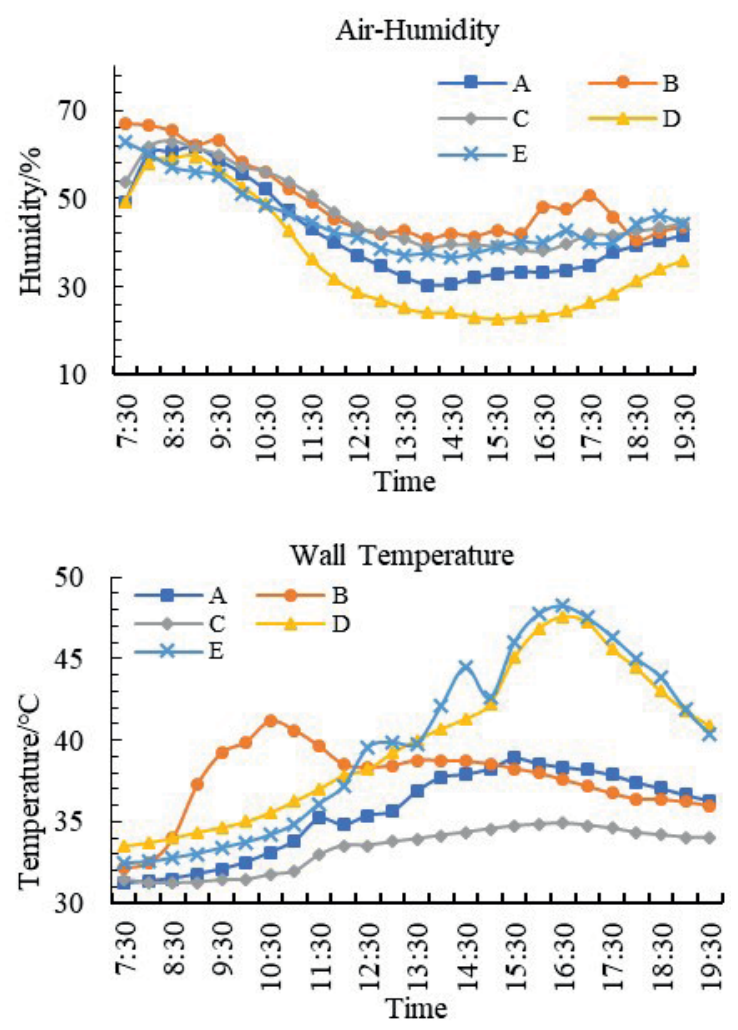

points appeared before 12:00 noon. Furthermore, the higher value of $\mathrm{T}_{\mathrm{mrt}}$ at point $\mathrm{C}$ lasted only about one hour.

The Bioklima software is used to calculate the Universal Thermal Climate Index (UTCI) through inputting data of air temperature, humidity, wind speed, average radiation temperature. The calculation results of each point are shown in Fig. 9. It can be seen that the UTCI values of the 5 measuring points are greatly distinctive. Temperatures of all points exceed the strong heat stress ( $32-38)$, therefore the thermal comfort is extremely poor. Similar to $\mathrm{T}_{\mathrm{mrt}}$, the maximum values of point $\mathrm{D}$ and $\mathrm{E}$ also appeare after 15:00, while the maximum values of the other three points appeare before 12:00 noon. Besides, except for point C, the UTCI values of other points in most of the measuring time exceed 38 (very strong heat stress[25]). Moreover, the values of point $\mathrm{D}$ belong to very strong heat stress in $72 \%$ of the testing time, and it is in the state of extreme health stress in $52 \%$ of the time.

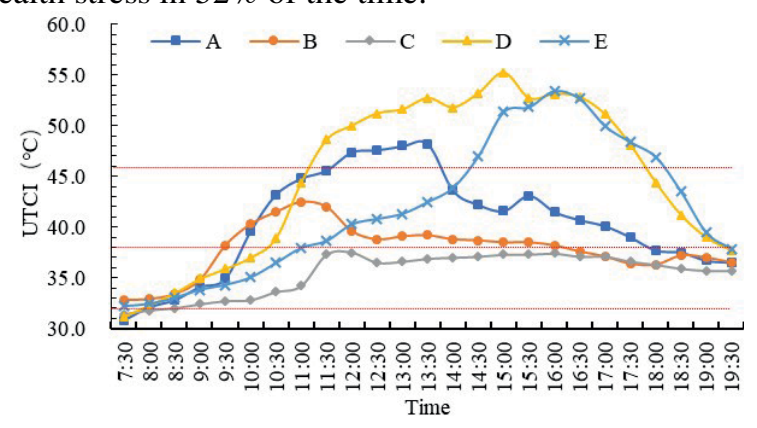

Fig. 9. UTCI of each measuring point

\footnotetext{
* Corresponding author: young30331@163.com
} 


\subsection{Analysis of factors influencing the thermal comfort evaluation of mountain city footpath}

The factors influencing the thermal comfort evaluation of mountain city footpath were analyzed respectively (Fig. 10, Fig. 11). The $\mathrm{R}^{2}$ value of 0.88 shows that the linear correlation between SVF and UTCI is distinctly large. While the linear correlation between $\mathrm{H} / \mathrm{W}$ is relatively weak, with the $\mathrm{R}^{2}$ value of only 0.04 . This indicates that the surrounding environment of the street, such as vegetation, has a great impact on thermal comfort evaluation. Thus, it is difficult and quite unreasonable to design a comfortable thermal environment only by taking $\mathrm{H} / \mathrm{W}$ into consideration.

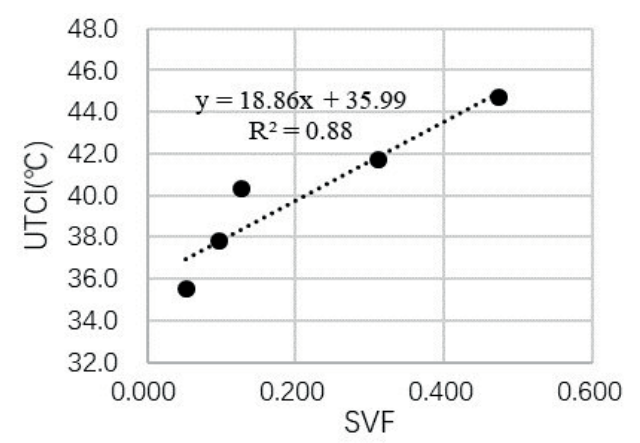

Fig. 10. The relationship between SVF and UTCI

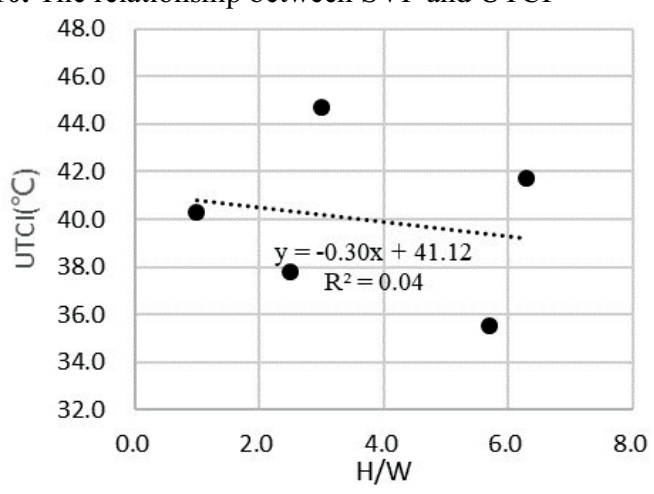

Fig. 11. The relationship between $\mathrm{H} / \mathrm{W}$ and UTCI

According to the interface analysis, five measuring points can be divided into two types: Bilateral encircling type and unilateral open type. It can be found that the UTCI value of unilateral open type is higher than that of bilateral encircling type from Table 2. At the same time, the UTCI near the mountain is 3 lower than that near the building, demonstrating that different interfaces have a great impact on the thermal comfort of the microenvironment.

Table 2. UTCI of Different interfaces

\begin{tabular}{cccc|cc}
\hline & \multicolumn{2}{c|}{ Bilateral encircling } & \multicolumn{2}{c}{ Unilateral open } \\
interface & $\mathrm{W}-\mathrm{B}$ & $\mathrm{B}_{1}-\mathrm{B}_{1}$ & $\mathrm{~B}_{2}-\mathrm{B}_{2}$ & $\mathrm{~B}-\mathrm{N}$ & $\mathrm{M}-\mathrm{N}$ \\
\hline UTCI ( & 40.3 & 37.8 & 35.5 & 44.7 & 41.7 \\
$\left.{ }^{\circ} \mathrm{C}\right)$ & 40.3 & 37.8 & & & \\
\hline
\end{tabular}

\section{Conclusions}

With huge quantities of greenhouse gases emission and rapid global climate change, urban climate problems are increasingly serious. Improving urban climate and creating a comfortable physical environment of the block is an urgent problem to be solved. Because this is an important measure to meet with people's expectation for a healthy and livable living environment. In this study, we measured the microclimate parameters of five different types of pedestrian streets in Chongqing and evaluated the outdoor thermal environment by UTCI. The relationship between microclimate and multi-factors was quantified. The conclusions can be drawn as follows: the sky view factor has a great influence on the thermal environment of the streets. The unilateral open space type B-N $(\mathrm{SVF}=0.474)$ has the worst thermal environment, which the average UTCI reaches as high as 44.7. On the contrary, the thermal environment of bilateral encircling space type $\mathrm{B}_{2}-\mathrm{B}_{2}(\mathrm{SVF}=0.052)$ is much better, with an average UTCI of 35.5 . Besides, the linear correlation between UTCI and SVF is larger, with the $\mathrm{R}^{2}=0.88$. While the linear correlation between $\mathrm{H} / \mathrm{W}$ is comparatively weak, with the $\mathrm{R}^{2}=0.04$. The surrounding environment of mountain city pedestrian streets, such as vegetation, has a great influence on thermal comfort evaluation. Different interfaces and enclosure form have a certain influence on thermal comfort. The UTCI value of unilateral open type is higher than that of bilateral encircling type, and the UTCI value of street near the mountain (M-N) is 3 lower than that of the street near buildings (B-N). Therefore, the corresponding environmental design should be taken into consideration in the reconstruction or design of mountain city footpath according to the regional environmental characteristics. The microclimate environment can also be improved from the perspective of green planting. This study can provide not only scientific guidance for the construction of ecological civilization in mountainous cities, but also comfort design experience and reference for the construction of livable and healthy living environment in future cities.

\section{References}

1. Jiaping Liu, et al., Urban environment physics ( China Architecture \& Building Press, 2011)

2. Junyan Yang, Xin Sun, Xing Shi, Coupling mechanism and optimal design of thermal environment and spatial form in urban center. (Southeast University Press, NanJing, 2016)

3. General Office of Chongqing Municipal People's Government, Opinions of General Office of Chongqing Municipal People's Government on strengthening the planning and implementation of historical and cultural protection and inheritance, (2019)

4. Potchter O, Cohen P, Lin T, et al., Sci. Total Environ. 631-632, 390-406 (2018) 
5. Hamdan D M A, de Oliveira F L., Energy Build. 200, 86-103 (2019)

6. Muniz-Gäal L P, Pezzuto C C, Carvalho M F H D, et al., Build. Environ. 169, 106547 (2020)

7. Mohajeri N, Gudmundsson A, Kunckler T, et al.,. Appl. Energy. 240, 173-190 (2019)

8. Nasir D S N M, Hughes B R, Calautit J K, et al., Energy Procedia 105, 4414-4419 (2017)

9. Shimazaki Y, Yoshida A, Suzuki R, et al., Build. Environ. 46, 1716-1724 (2011)

10. Bourbia F, Boucheriba F, Renew. Energy. 35, 343347 (2010).

11. Shaohua Tan, Yawen Sun, Jize Shen, Urbanism and Architecture 24, 24-28 (2018)

12. Shaohua Tan, Ling Han, Urban problems 02, 43-49 (2015)

13. Yubo Li, Shaohua Tan, Architecture\&Culture 08, 90-91 (2018)

14. Feidong Lu, Shaohua Tan, Planners 35, 69-76 (2019)

15. Guifeng Han, Zhi Cai, Yusi Xie, et al., Civil and environmental engineering 38, 138-147 (2016)

16. Guifeng Han, Wentao Yan, Ke Zhao, et al., Research of Environmental Sciences 25, 615-621 (2012)

17. Guifeng Han, Ke Zhao, Wentao Yan,et al., Chinese Journal of Applied Ecology 23, 1655-1662 (2012)

18. Guifeng Han, Zhi Cai, Journal of Human Settlements in West China 31, 61-68 (2016)

19. Guifeng Han, Lin Ye, Zhongwei Sun, Acta Ecologica Sinica 34, 4017-4024 (2014)

20. Zhi Cai, Guifeng Han, Mountain Research 36, 617 627 (2018)

21. Jianhua Zhou, Chongqing University (2010)

22. Matzarakis A, Rutz F, Mayer H, Int. J. Biometeorol. 54, 131-139 (2010)

23. Matzarakis A, Rutz F, Mayer H, Int. J. Biometeorol. 51, 323-334 (2007)

24. Thorsson S, Lindberg F, Eliasson I, et al., Int. J. Climatol. 27, 1983-1993 (2007)

25. Kampmann B, De P B, Fiala D, Int. J. Biometeorol. 56, 505-513 (2012) 